\section{Poluição atmosférica e internações hospitalares por doenças respiratórias em crianças e adolescentes em Cubatão, São Paulo, Brasil, entre 1997 e 2004}

\author{
Air pollution and pediatric hospital admissions due \\ to respiratory diseases in Cubatão, São Paulo \\ State, Brazil, from 1997 to 2004
}

\author{
1 Programa de Pós-graduação \\ de Saúde Coletiva, \\ Universidade Católica de \\ Santos, Santos, Brasil. \\ Correspondência \\ R. Jasinki \\ Grupo de Avaliação de \\ Exposição e Risco Ambiental, \\ Programa de Pós-graduação \\ de Saúde Coletiva, \\ Universidade Católica de \\ Santos. \\ Rua Saturnino de Brito 166, \\ apto. 28, Santos, SP \\ 11070-001, Brasil. \\ re_jasinski@yahoo.com.br
}

\begin{abstract}
The aim of this study was to evaluate the effects of air pollutants and their lag structures in relation to respiratory morbidity among children and adolescents in the city of Cubatão, São Paulo State, Brazil, from 1997 to 2004. An ecological time-series study was performed, analyzing respiratory hospital admissions of children and adolescents in National Health System hospitals in Cubatão. Generalized linear Poisson regression models were used to control for seasonality, temperature, humidity, and short-term trends. $P M_{10}$ and ozone were significantly associated with respiratory hospital admissions. Among children, inter-quartile range increases in the PM ${ }_{10} 7$-day moving average $\left(56.5 \mu \mathrm{g} / \mathrm{m}^{3}\right)$ and in the ozone 5-day moving average $\left(46.7 \mu \mathrm{g} / \mathrm{m}^{3}\right)$ led to increases of 9.6\% (95\%CI: 3.0\%-16.1\%) and 2.4\% (95\%CI: 0.1\%-4.7\%) in respiratory hospital admissions, respectively. Efforts to reduce air pollutants need to be adopted to minimize the adverse effects on children and adolescents in Cubatão.
\end{abstract}

Air Pollution; Respiratory Tract Diseases; Hospitalization; Child; Adolescent
Renata Jasinski 1

Luiz Alberto Amador Pereira 1

Alfésio Luís Ferreira Braga 1

\section{Introdução}

Desde a Revolução Industrial, a poluição atmosférica tem se tornado um fator de risco extremamente importante para a saúde humana, especialmente nos centros urbanos industrializados. A partir de meados do século XX, medidas passaram a ser adotadas, em diferentes países, para controle das emissões de poluentes atmosféricos, objetivando, inicialmente, a redução de episódios com excessiva concentração de poluentes. Os padrões de qualidade do ar adotados a princípio estabeleciam níveis de concentração de poluentes mais elevados. Entretanto, com o aprimoramento dos recursos de investigação epidemiológica, observa-se que mesmo com concentrações abaixo do limite de segurança estabelecido pela legislação vigente, os poluentes atmosféricos podem acarretar efeitos deletérios à saúde humana 1,2,3.

Em termos amplos, os estudos nessa área podem ser separados entre aqueles que investigam os efeitos agudos $3,4,5,6,7,8$ e os que investigam os efeitos crônicos da exposição à poluição do ar $9,10,11$. O primeiro é mais numeroso e pesquisa desfechos como morbimortalidade, sintomatologia, hospitalizações e atendimentos em emergência, associados às concentrações de poluentes atmosféricos, num estudo de base populacional agregada de série de tempo, do qual se trata o presente. $\mathrm{O}$ segundo, denominado estudo de 
coorte, envolve análise de dados que necessitam de um determinado tempo de seguimento como, por exemplo, mortalidade total sobre uma doença com os efeitos da exposição prolongada à poluição ambiental.

Além disso, diversos estudos permitem afirmar que as faixas etárias mais susceptíveis são as crianças 4,5,7,12,13,14 e os idosos 6,15,16,17,18. Os indivíduos portadores de doenças preexistentes, como as cardiopatias 19,20,21 e as pneumopatias 22, também são mais suscetíveis aos efeitos dos poluentes atmosféricos.

As principais fontes de emissão associadas à contaminação do ar são as indústrias e os veículos automotores. Cubatão, o lugar em estudo, é uma cidade industrial no Estado de São Paulo com crescimento contínuo em sua frota automotiva. Na década de 80, um incêndio na Vila Socó (1984) e o vazamento de amônia com evacuação da Vila Parisi (1985) obrigaram as autoridades a adotar políticas públicas mais restritivas para emissão de poluentes, o que levou à redução na emissão de poluentes 23 .

Este estudo tem por objetivo analisar os efeitos dos poluentes atmosféricos sobre as crianças e adolescentes residentes na cidade de Cubatão durante o período de transição do final do século XX para os primeiros anos do século XXI com a utilização de dados secundários de internações hospitalares por doenças respiratórias.

\section{Material e métodos}

Foram coletados dados de internações hospitalares por doenças respiratórias de moradores de Cubatão, fornecidos pelo Departamento de Informática do Sistema Único de Saúde (DATASUS), para o período de 1997 a 2004. Foram selecionadas todas as internações por doenças respiratórias (CID-9a revisão: 620-519; CID-10a revisão: J00 ao J99) de crianças e adolescentes (0 a 19 anos) moradores da cidade de Cubatão, que ocorreram entre os anos de 1997 e 2004, nos hospitais da cidade.

As concentrações dos poluentes do ar $\left(\mathrm{PM}_{10}\right.$, $\mathrm{NO}_{2}, \mathrm{SO}_{2} \mathrm{e} \mathrm{O}_{3}$ ) foram fornecidas pela Companhia de Tecnologia e Saneamento Ambiental do Estado de São Paulo (CETESB), juntamente com os valores diários de temperatura mínima e umidade relativa do ar. A CETESB dispõe de duas estações fixas de monitoramento na cidade de Cubatão. Os registros para os poluentes $\mathrm{PM}_{10}$ e $\mathrm{SO}_{2}$ foram obtidos das duas estações de monitoramento (Centro e Vila Parisi) enquanto o $\mathrm{NO}_{2}$ e $\mathrm{O}_{3}$ foram registrados apenas na estação do Centro. As redes de monitoramento da região não registraram os níveis diários do CO, razão pela qual este poluente não será incluído na pesquisa.

Para estimar os efeitos da variação diária na concentração dos poluentes sobre os desfechos de interesse, foram utilizados modelos lineares generalizados de regressão de Poisson específicos para cada grupo etário, a saber, 0 a 5 anos, 6 a 10 anos, 0 a 10 anos e de 11 a 19 anos, controlados para sazonalidade, temperatura mínima, umidade relativa e dia da semana 24 .

A relação não linear entre a sazonalidade e os desfechos de interesse (internações hospitalares) foi controlada por meio de funções de alisamento semiparamétricas (natural cubic spline) 25. Além da sazonalidade, os estudos realizados com atendimentos de pronto-socorro e internações hospitalares mostram que existe um padrão de variação de curta duração influenciando a relação entre poluição atmosférica e saúde. Dias da semana precisam ser controlados nos modelos de regressão, sobretudo para desfechos relacionados às admissões hospitalares. Para isso, foram criados indicadores para dias da semana e para os feriados 26 .

Para reduzir a sensibilidade da variável dependente a valores extremos da variável independente, foi utilizado o método de regressão robusta (estimador-M).

Os efeitos dos poluentes atmosféricos nos desfechos escolhidos foram investigados pela utilização de defasagens das concentrações dos poluentes em modelos com um único poluente. Efeitos acumulados foram estimados com a utilização de médias móveis das concentrações diárias dos poluentes.

Os resultados foram expressos em aumento percentual nas admissões hospitalares e respectivos intervalos de 95\% de confiança (IC95\%), por conta de aumentos de um interquartil (a diferença entre o terceiro e o primeiro quartis) nas concentrações dos poluentes.

Os bancos de dados foram preparados com o uso do programa Statistical Package for Social Science (SPSS) para Windows, versão 13.0 (SPSS Inc., Chicago, Estados Unidos). As análises de regressão foram feitas com o programa S-PLUS 4.5 (Mathsoft Inc., Seattle, Estados Unidos).

\section{Resultados}

Ocorreram, durante o período de estudo, 6.630 internações de crianças e adolescentes por doenças respiratórias. A faixa etária de 0 a 10 anos representou 95\% dos casos (6.291), sendo a maioria absoluta deles de crianças com 5 anos ou menos (5.671). Os cinco por cento restantes foram formados por adolescentes de 11 a 19 anos (339). A 
Tabela 1 apresenta a análise descritiva das variáveis (faixa etária, poluentes e variáveis meteorológicas) utilizadas neste estudo.

Pode-se observar que as internações mais frequentes foram na faixa etária de 0 a 10 anos de idade (6.291 casos) e o menor número de eventos ocorreu entre 11 a 19 anos.

Ao longo dos 2.922 dias do estudo, o $\mathrm{PM}_{10}$ foi medido em 2.722 no Centro e em 2.896 dias na Vila Parisi. As médias diárias de $\mathrm{PM}_{10}$ apresentaram diferenças evidentes entre as estações do Centro e da Vila Parisi. Na última, a média desse poluente foi 2,4 vezes maior do que a observada na estação de monitoramento localizada no centro da cidade e ultrapassou o padrão de qualidade do ar para o poluente $\left(50 \mu \mathrm{g} / \mathrm{m}^{3}\right.$ de média anual). Na região central, ocorreram ultrapassagens do valor de máxima diária $\left(150 \mu \mathrm{g} / \mathrm{m}^{3}\right)$ em apenas dez dias durante os oito anos avaliados. Na região de Vila Parisi, as ultrapassagens do padrão foram frequentes e sem respeitar a sazonalidade. As concentrações diárias de $\mathrm{PM}_{10}$ registradas no Centro e na Vila Parisi apresentaram correlação de Pearson de 0,60 ( $p<0,01)$.

Foram feitas medidas de $\mathrm{SO}_{2}$ no Centro em 2.200 dias e na Vila Parisi em 2.398 dias. Em relação ao $\mathrm{SO}_{2}$, a estação de Vila Parisi registrou a maior média quando comparada à estação do Centro. Contudo, a diferença entre as médias registradas pelas duas estações foi muito menor do que a encontrada para as medidas de $\mathrm{PM}_{10}$. Além disso, as duas medidas de $\mathrm{SO}_{2}$ ficaram abaixo do padrão de qualidade para tal poluente $(80 \mu \mathrm{g} /$ $\mathrm{m}^{3}$ de média anual). Não ocorreram ultrapassagens do valor máximo diário para este poluente $\left(365 \mu \mathrm{g} / \mathrm{m}^{3}\right)$ ao longo do período de estudo nas duas estações de monitoramento. As concentrações diárias de $\mathrm{SO}_{2}$ registradas no Centro e na Vila Parisi apresentaram correlação de Pearson de $0,33(\mathrm{p}<0,01)$.

As concentrações do $\mathrm{NO}_{2}$, medido em apenas uma estação (Centro) em 2.172 dias, estiveram abaixo do padrão adotado $\left(100 \mu \mathrm{g} / \mathrm{m}^{3}\right.$ de média anual). Os valores diários também estiveram aquém do valor máximo tolerado para este poluente, que é de $320 \mu \mathrm{g} / \mathrm{m}^{3}$.

O ozônio, medido apenas na estação Centro, apresentou registros válidos em 2.610 dias. Ao longo do período, ocorreram ultrapassagens do valor horário máximo $\left(160 \mu \mathrm{g} / \mathrm{m}^{3}\right)$ em $6 \%$ dos dias.

A Figura 1 apresenta as estimativas de aumento percentual nas internações por doenças respiratórias em crianças com menos de seis

\begin{tabular}{|c|c|c|c|c|c|c|c|}
\hline & Média & DP & Mínimo & 1o quartil & 3o quartil & Máximo & $\begin{array}{l}\text { Ultrapassa- } \\
\text { gens diárias }\end{array}$ \\
\hline \multicolumn{8}{|c|}{ Internações respiratórias por faixas } \\
\hline \multicolumn{8}{|c|}{ etárias (anos) } \\
\hline $0-5$ & 1,9 & 1,7 & 0 & 1 & 3 & 18 & \\
\hline $6-10$ & 0,2 & 0,5 & 0 & 0 & 0 & 4 & \\
\hline $0-10$ & 2,1 & 1,8 & 0 & 1 & 3 & 18 & \\
\hline $11-19$ & 0,1 & 0,4 & 0 & 0 & 0 & 3 & \\
\hline \multicolumn{8}{|l|}{ Poluentes } \\
\hline Centro - $\mathrm{PM}_{10}\left(\mu \mathrm{g} / \mathrm{m}^{3}\right)$ & 36,4 & 19,3 & 5,2 & 24,6 & 44 & 259,6 & $10 x$ \\
\hline Vila Parisi - $\mathrm{PM}_{10}\left(\mu \mathrm{g} / \mathrm{m}^{3}\right)$ & 85,8 & 46,4 & 8,0 & 59,8 & 116,4 & 614,6 & $288 x$ \\
\hline $\mathrm{PM}_{10}$ médio $\left(\mu \mathrm{g} / \mathrm{m}^{3}\right)$ & 63,8 & 30,0 & 2,83 & 43,4 & 79,5 & 325,4 & - \\
\hline Centro $-\mathrm{SO}_{2}\left(\mu \mathrm{g} / \mathrm{m}^{3}\right)$ & 15,8 & 12,7 & 1,0 & 9,3 & 22,6 & 128,5 & - \\
\hline Vila Parisi $-\mathrm{SO}_{2}\left(\mu \mathrm{g} / \mathrm{m}^{3}\right)$ & 24,5 & 20,1 & 1,0 & 10,5 & 32,2 & 183,3 & - \\
\hline $\mathrm{NO}_{2}\left(\mu \mathrm{g} / \mathrm{m}^{3}\right)$ & 26,7 & 12,1 & 2,9 & 18,4 & 32,7 & 117 & - \\
\hline $\mathrm{O}_{3}\left(\mu \mathrm{g} / \mathrm{m}^{3}\right)$ & 87,4 & 74,6 & 5,5 & 56,5 & 103,2 & 686 & $167 x$ \\
\hline \multicolumn{8}{|l|}{ Variáveis meteorológicas } \\
\hline Temperatura $\left({ }^{\circ} \mathrm{C}\right)$ & 20 & 3,5 & 0 & 17,5 & 22,7 & 42 & \\
\hline Umidade (\%) & 90,6 & 9,3 & 16,6 & 85,3 & 95,2 & 99,4 & \\
\hline
\end{tabular}

DP: desvio-padrão. 
Aumento percentual nas internações por doenças respiratórias de crianças com até 5 anos de idade em razão de um aumento de um interquartil $\left(36,1 \mu \mathrm{g} / \mathrm{m}^{3}\right)$ na concentração de $\mathrm{PM}_{10}$ médio. Cubatão, São Paulo, Brasil.

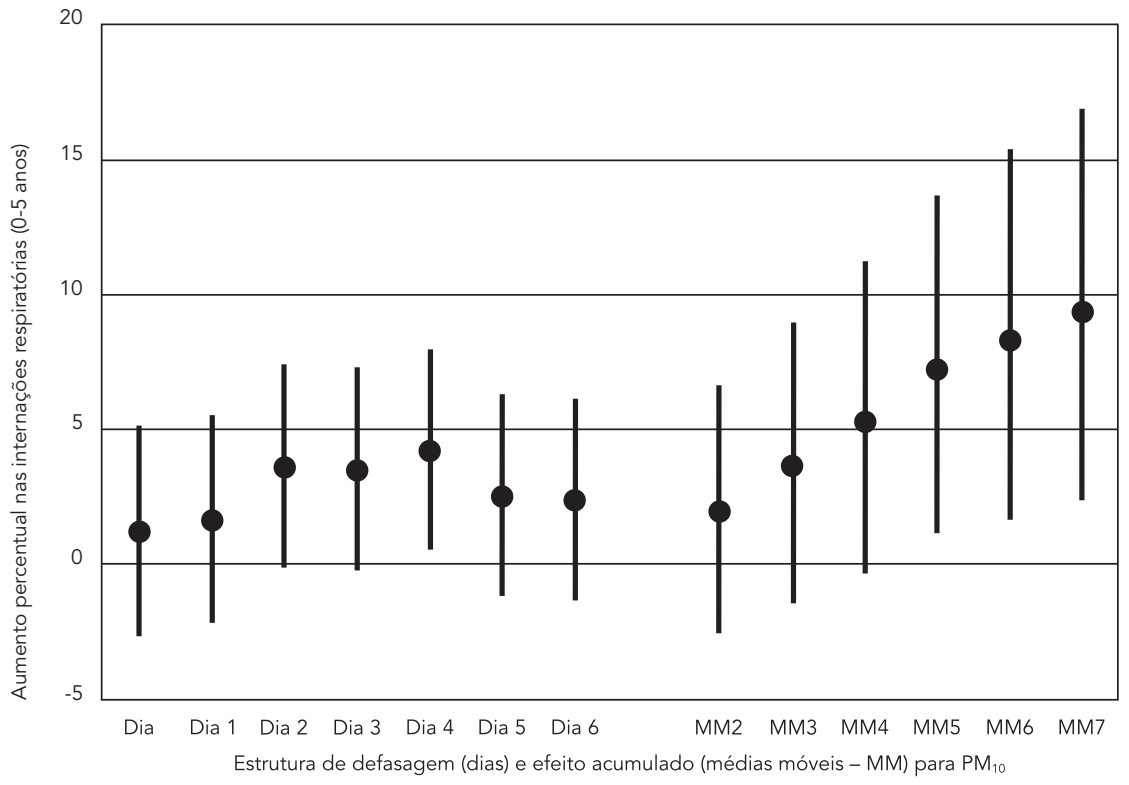

anos de idade em razão de aumentos de um interquartil nas concentrações diárias de $\mathrm{PM}_{10}$ médio (média dos valores registrados no Centro e na Vila Parisi).

$\mathrm{Na}$ análise da estrutura de defasagem, verifica-se um efeito sempre positivo que se torna estatisticamente significativo no segundo dia após o aumento do poluente e que assim permanece por três dias. Até seis dias após o aumento do poluente, pode-se chegar a um aumento de $10 \%$ nas internações de crianças nessa faixa etária.

Quando as análises foram feitas com as concentrações específicas de cada uma das estações de monitoramento, constatou-se que o padrão de defasagem era mais longo para o $\mathrm{PM}_{10}$ medido na Vila Parisi do que o registrado na estação Centro. As estimativas de efeito acumulado apresentaram magnitudes diferentes de acordo com o local de monitoramento do poluente, reflexo direto de diferenças nas concentrações registradas nas duas áreas analisadas. Adotou-se a estimativa feita com o $\mathrm{PM}_{10}$ médio para representar o efeito esperado do poluente no desfecho indicado.

As análises dos efeitos de $\mathrm{PM}_{10}$ nas internações de crianças de 6 a 10 anos de idade por doenças respiratórias não apresentaram efeitos estatisticamente significativos e nem um padrão de estrutura de defasagem definido.

Quando foram agrupadas as crianças menores de 10 anos em um único grupo, observou-se um padrão de efeito bastante semelhante ao encontrado para as crianças com cinco anos ou menos para variações nas concentrações do $\mathrm{PM}_{10}$ médio (Figura 2).

Todavia, as magnitudes do efeito acumulado e dos dias ao longo da estrutura de defasagem foram menores, pois apenas as estimativas com o $\mathrm{PM}_{10}$ da Vila Parisi apresentaram efeitos estatisticamente significativos.

Os efeitos do $\mathrm{SO}_{2}$ e $\mathrm{NO}_{2}$ sobre as internações respiratórias foram menores do que os observados para o $\mathrm{PM}_{10}$ e não impactaram de modo relevante na faixa etária em estudo.

$\mathrm{O} \mathrm{O}_{3}$ foi medido apenas na região Central da cidade. Portanto, suas estimativas de efeito estão relacionadas aos valores medidos em apenas uma estação de monitoramento.

Em relação ao $\mathrm{O}_{3}$, para crianças e adolescentes, as estruturas de defasagem apresentaram padrões semelhantes, com efeito agudo (dia da exposição) e perda do efeito no dia seguinte. Para 
Aumento percentual nas internações por doenças respiratórias de crianças com até 10 anos de idade em razão de um aumento de um interquartil $\left(36,1 \mu \mathrm{g} / \mathrm{m}^{3}\right)$ na concentração de PM $_{10}$ médio. Cubatão, São Paulo, Brasil.

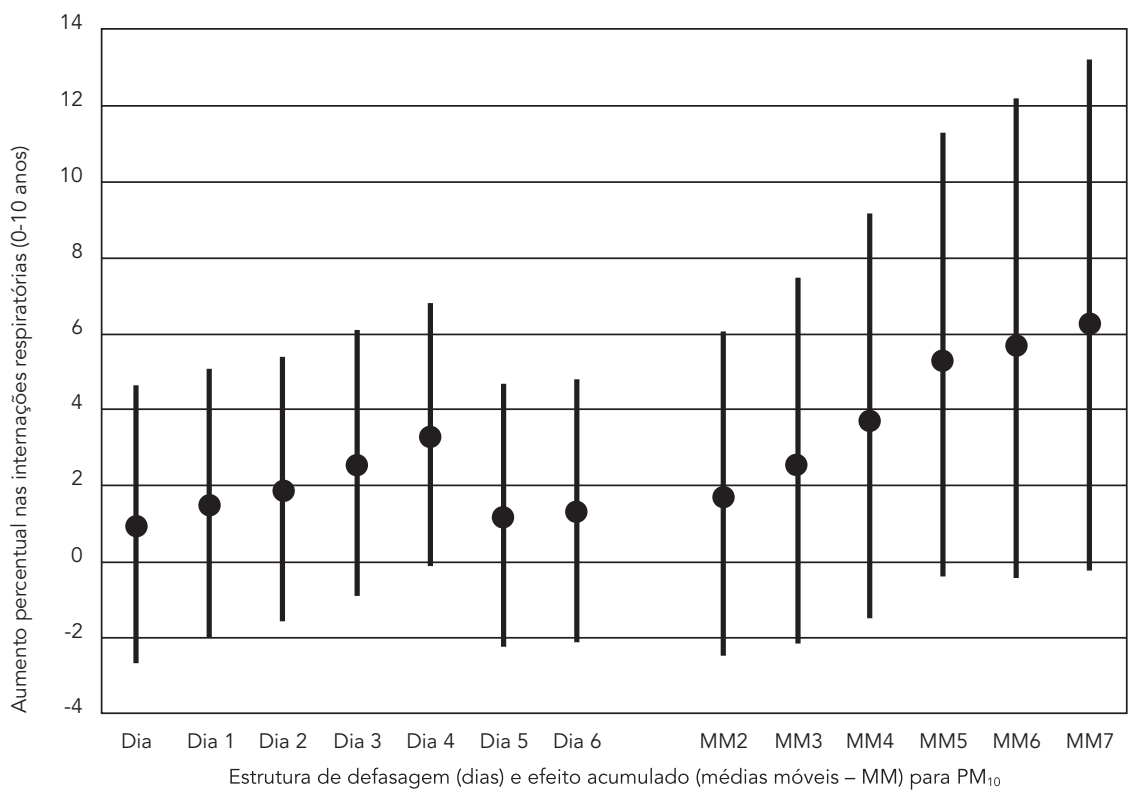

as crianças até dez anos de idade ainda foram percebidos efeitos acumulados de até cinco dias (Figuras 3 e 4 ).

Entre os adolescentes, verificou-se efeito apenas no dia da exposição e com maior magnitude (6,7\%, IC95\%: 0,04-13,9) quando comparado ao efeito nas crianças.

\section{Discussão}

Este estudo mostrou que na cidade de Cubatão, entre a segunda metade da década de 1990 até 2004, apesar de haver uma redução nos níveis de poluição atmosférica desde o início de seu monitoramento, os poluentes analisados $\left(\mathrm{PM}_{10}\right.$, $\mathrm{SO}_{2}, \mathrm{NO}_{2}$ e $\mathrm{O}_{3}$ ) apresentaram efeitos relevantes sobre a saúde dos moradores da cidade. Tal efeito foi avaliado pelas internações hospitalares por doenças respiratórias em crianças e adolescentes.

Os efeitos encontrados resistiram à inclusão das variáveis de confusão classicamente utilizadas em estudos semelhantes: tendências de longa (sazonalidade) e curta (dias da semana) duração e as variáveis meteorológicas. Tem sido demonstrado por outros autores que a exclusão de características ou variáveis que não apresentam periodicidades concordantes com a variável dependente e com as variáveis independentes não introduz nas estimativas um viés sistemático e, portanto, não comprometem o estudo 27 . Mesmo com toda a precaução adotada na escolha das variáveis de confusão incluídas nos modelos, não se pode excluir, definitivamente, a omissão de alguma delas, ainda que isso seja altamente improvável.

Indicadores de uso de serviços de saúde só podem ser referidos a uma população quando o local de moradia dos usuários é conhecido. $\mathrm{O}$ local de moradia dos usuários dos serviços é um dado fundamental para a análise do fluxo de pacientes entre municípios e distritos sanitários, informação importante para o planejamento/programação da oferta de serviços de saúde e para a alocação de recursos financeiros 28 . No banco de dados obtido junto ao DATASUS, as internações foram selecionadas pelo local de moradia, ou seja, moradores da cidade de Cubatão, e não por localização dos hospitais. Logo, morador da cidade que internou em cidade vizinha também foi incluído na pesquisa. 
Figura 3

Aumento percentual nas internações por doenças respiratórias de crianças com até 5 anos de idade em razão de um aumento de um interquartil $\left(46,7 \mu \mathrm{g} / \mathrm{m}^{3}\right)$ na concentração de $\mathrm{O}_{3}$. Cubatão, São Paulo, Brasil.

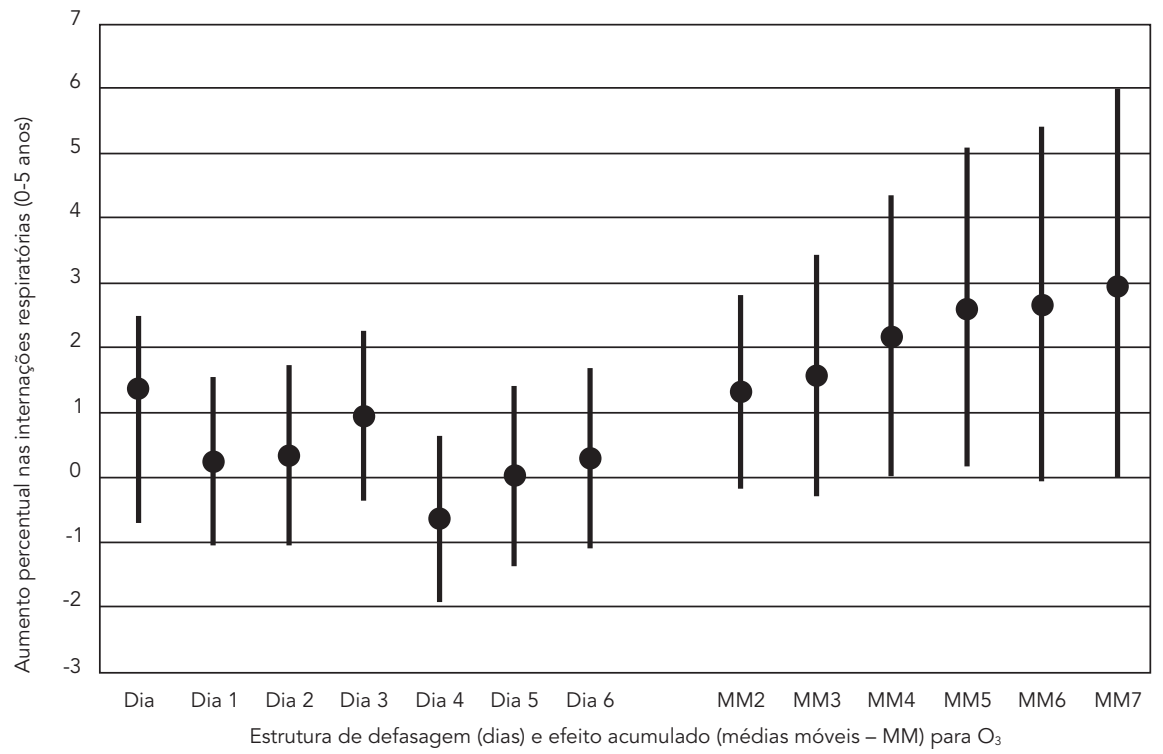

Figura 4

Aumento percentual nas internações por doenças respiratórias de crianças com até 10 anos de idade em razão de um aumento de um interquartil $\left(46,7 \mu \mathrm{g} / \mathrm{m}^{3}\right)$ na concentração de $\mathrm{O}_{3}$. Cubatão, São Paulo, Brasil.

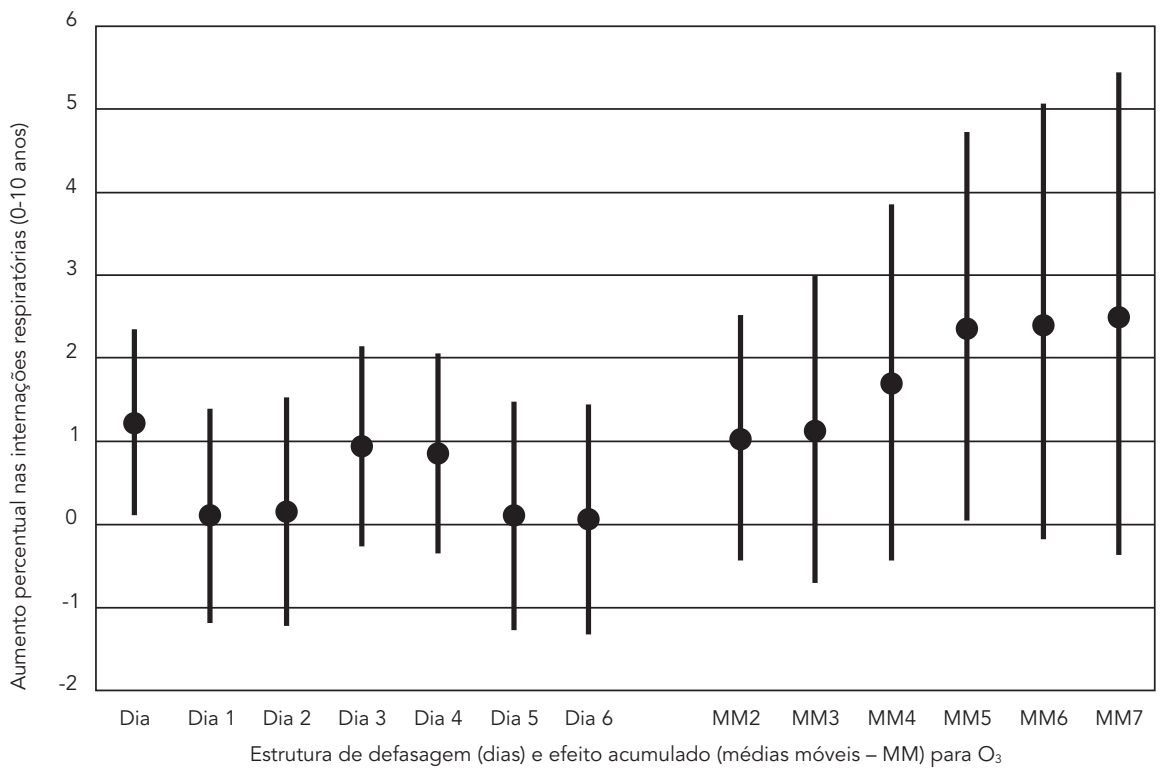


Estudo de Veras \& Martins 28, comparando as informações extraídas do sistema de arquivos eletrônicos com os registros de informações de cada paciente avaliado em determinados hospitais, concluíram que ocorreu considerável acordo entre as informações, especialmente para o diagnóstico e variáveis demográficas. No entanto, no presente estudo, foi utilizada a doença respiratória como causa de internação hospitalar e não patologias respiratórias específicas, na tentativa de reduzir o erro de classificação de doença, uma vez que os dados do SIH/ SUS foram obtidos em ambientes hospitalares diversificados.

Os dados de qualidade do ar utilizados no estudo, valores diários dos poluentes medidos pela CETESB entre 1997 e 2004, podem ser considerados de boa qualidade, já que a companhia tem experiência no tema e tem sido parceira de diferentes pesquisadores brasileiros em estudos de contaminação atmosférica e efeitos na saúde nos últimos 20 anos. Contudo, dois fatos podem ser lamentados: a ausência de dados de CO para o período estudado e a falta de medidas realizadas em mais de um ponto para $\mathrm{NO}_{2} \mathrm{e} \mathrm{O}_{3}$. A topografia da região, as correntes de vento, a distribuição irregular da população e das indústrias pela área podem criar microambientes com características peculiares e consequente diferenciação na exposição e nos efeitos verificados. A presença de mais monitores que registrem todos os poluentes primários e o ozônio poderia melhorar a análise da qualidade do ar nos diferentes nichos geográficos e populacionais da região. Por outro lado, a diversidade de fontes fixas e móveis e a sua distribuição por uma área ampla que adentra a região urbana, além da contribuição mais recente e crescente das fontes móveis permitem que se assuma uma exposição relativamente homogênea.

Os estudos que utilizam dados do SUS apresentam uma limitação inerente ao próprio sistema: excluem dados dos hospitais particulares que não estão conveniados ao SUS. As informações sobre internações de pacientes com planos de saúde privados, apesar de registradas na Secretaria Estadual de Saúde, não estavam disponíveis. Desse modo, generalização dos resultados deverá ser feita para populações que apresentem idênticas características daquela atendida pelos hospitais conveniados ao SUS e que habitem áreas onde a complexidade da mistura de poluentes do ar de diferentes fontes se assemelhe à encontrada na região de Cubatão. Para o período estudado, as informações referentes ao endereço de moradia dos pacientes internados não apresentava consistência de registro que permitisse sua utilização e análise da distribuição espacial dos casos ou uma melhor caracterização da exposição individual.

A existência de associação entre morbidade respiratória e níveis de poluição atmosférica já foi detectada e comprovada em vários estudos realizados no Brasil 6,7,8,19,29,30,31 e no mundo $20,32,33$.

Outros estudos já mencionados vêm mostrando que mesmo em baixas concentrações a poluição atmosférica está associada com efeitos na saúde 13. Contudo, questões como qual ou quais poluentes têm associação mais robusta com os efeitos à saúde ainda não estão esclarecidas. A tentativa de isolar os efeitos individuais dos diferentes poluentes é uma tarefa difícil devida, sobretudo, à correlação positiva usualmente presente entre os poluentes primários.

A maior parte dos estudos que avalia os efeitos dos poluentes do ar sobre as internações hospitalares por doenças respiratórias foi realizada na década de 1990. Desde então o interesse passou a ser maior em relação aos efeitos cardiovasculares dos poluentes atmosféricos. Entretanto, os estudos mais recentes que utilizam as doenças respiratórias como um indicador dos efeitos adversos dos contaminantes do ar mostram que o tema está longe de se esgotar.

De acordo com os resultados da presente análise, a morbidade respiratória para crianças e adolescentes constatou associação positiva com $\mathrm{PM}_{10}$ e $\mathrm{O}_{3}$. Ocorreu um incremento de aproximadamente $10 \%$ nas internações por doenças respiratórias para menores de cinco anos para $\mathrm{PM}_{10}$ médio, para aumento de um interquartil na concentração do poluente até seis dias após a exposição. O padrão de defasagem foi mais longo e o efeito de maior magnitude para a Vila Parisi em relação ao Centro. Esse fato pode ser causado pelas diferenças nas concentrações entre as duas áreas e/ou diferença na composição das partículas. $\mathrm{O} \mathrm{O}_{3}$ apresentou efeito agudo para as crianças e adolescentes, no dia da exposição, com acréscimos de 1,3\% e 6,8\%, respectivamente, para um incremento de uma variação interquartil na concentração diária do poluente.

Estudo realizado no início da década de 1990, na cidade de São Paulo, quando os níveis de poluentes atmosféricos eram maiores dos que os atuais, mostrou que uma variação de $70 \mu \mathrm{g} / \mathrm{m}^{3}$ na concentração diária de $\mathrm{PM}_{10}$ esteve associada com um aumento de $12 \%$ nas internações hospitalares por doenças respiratórias 27. Caso se utilize a mesma variação do estudo de Braga 27 para o efeito estimado em Cubatão para crianças, obter-se-ia um aumento de, aproximadamente, $20 \%$ nas internações. Isso evidencia o fato de a poluição em Cubatão, entre 1997 e 2004, ser mais associada às internações por doenças respirató- 
rias do que em São Paulo, no início da década de 1990. Em uma avaliação estratificada por faixas etárias com dados do período entre 1993 e 1997 na cidade de São Paulo, Braga et al. 5 mostraram que as crianças com até 2 anos de idade formam o grupo mais susceptível aos efeitos do $\mathrm{PM}_{10}$. Em relação ao $\mathrm{O}_{3}$, ele se mostrou associado apenas com efeitos adversos entre as crianças menores de 3 anos, sem afetar os adolescentes. Esse comportamento difere do encontrado em Cubatão provavelmente porque os níveis de $\mathrm{O}_{3}$ durante o presente estudo eram muito maiores do que os observados em São Paulo entre 1993 e 199734. Este estudo reforça a idéia de que apesar de as internações por doenças respiratórias não serem muito frequentes entre os adolescentes, eles são mais sensíveis às variações nas concentrações de poluentes atmosféricos do que as crianças entre 6 e 10 anos. A maior exposição aos ambientes externos, alterações hormonais que levem a uma redução temporária do sistema imune e tabagismo, por exemplo, podem estar relacionados a esta suscetibilidade 35 .

Estudos realizados com dados do período entre 1992 e 2000 na cidade de São Paulo mostram que os efeitos do $\mathrm{PM}_{10}$ e $\mathrm{O}_{3}$ sobre as crianças são consistentes ao longo das séries de tempo analisadas $30,34,36$. Farhat et al. 37 mostraram que o $\mathrm{O}_{3}$, assim como o $\mathrm{SO}_{2}$ e o $\mathrm{CO}$ estão associados com internações tanto de doenças respiratórias infecciosas quanto inflamatórias entre crianças e adolescentes.

Estudo realizado em Toronto, Canadá, evidenciou associação do $\mathrm{O}_{3}$ e problemas respiratórios em crianças menores de 2 anos, gerando aumento de até 35\% (IC95\%: 19\%-52\%) nas internações na média móveis de 5 dias para aumento de 45 ppb no valor máximo de $1 \mathrm{~h}$ de $\mathrm{O}_{3}$ nos meses quentes 38 . Lin et al. ${ }^{39}$, quatro anos após o estudo de Burnett 38 , reforçam os achados mostrando que níveis baixos de concentração de partículas inaláveis e poluentes gasosos, em Toronto, estavam associados com internações hospitalares de crianças e adolescentes.

Lin et al. 40 encontrou associações positivas entre as internações respiratórias e aumento dos níveis $\mathrm{O}_{3}$ dois dias antes da internação hospitalar. Aplicação de diferentes métodos estatísticos e análise de sensibilidade não alteraram essas descobertas.

Neste trabalho, foram verificadas características peculiares que divergem de outras publicações no que se refere à estrutura de defasagem do $\mathrm{PM}_{10}$. Para a faixa etária até cinco anos, encontrou-se, aqui, um efeito mais tardio com defasagem mais longa especialmente para o $\mathrm{PM}_{10}$ médio e o registrado na Vila Parisi (com efeito no 4o dia após exposição ao poluente). Tal peculiaridade do estudo em Cubatão pode ser consequência de alguns fatores associados. A possível dificuldade de acesso ao sistema de saúde induz os responsáveis pela criança à introdução de cuidados paliativos, postergando a procura pelo atendimento médico. Além disso, o efeito do $\mathrm{PM}_{10}$ pode ocorrer, prioritariamente, sobre as doenças infecciosas do trato respiratório 41,42 , doenças cuja manifestação pode se dar em um período mais tardio quando comparadas às exacerbações dos quadros puramente inflamatórios 43 .

Este estudo mostrou que durante o período avaliado, janeiro de 1997 a dezembro de 2004, os poluentes atmosféricos na cidade de Cubatão estiveram associados à morbidade respiratória, avaliada por intermédio dos números diários de internações hospitalares, de crianças e adolescentes. Entre crianças de 0 a 10 anos, foram observados efeitos para $\mathrm{PM}_{10}$ e $\mathrm{O}_{3}$ com estrutura de defasagem de 2 a 4 dias para as partículas enquanto para o poluente gasoso esse efeito foi mais relevante no dia da exposição, mas com efeito acumulado de cinco dias. Os efeitos sobre os adolescentes foram verificados apenas para a exposição aguda ao ozônio.

Apesar dos esforços para reduzir os níveis de emissão de poluentes ao longo das últimas décadas na região da cidade de Cubatão, as concentrações dos poluentes encontradas durante o período de estudo mostram que ainda são mensuráveis e significativos os efeitos da poluição atmosférica sobre a saúde respiratória de crianças e adolescentes. Por conseguinte, sugere-se que os esforços sejam intensificados na busca da menor concentração possível a fim de minimizar os efeitos adversos da exposição aos poluentes do ar em uma área com grande concentração de indústria e crescente participação automotiva. 


\section{Resumo}

O objetivo deste estudo foi avaliar os efeitos dos poluentes atmosféricos e suas estruturas de defasagem sobre a morbidade respiratória de crianças e adolescentes, na cidade de Cubatão, São Paulo, Brasil, entre 1997 e 2004. Um estudo ecológico de séries temporais foi realizado, analisando internações nos hospitais do Sistema Único de Saúde por doenças respiratórias de crianças e adolescentes residentes no município. Foram utilizados modelos lineares generalizados de regressão de Poisson, controlando-se para sazonalidade, temperatura, umidade e tendência de curta duração. $P M_{10}$ e ozônio estiveram positiva e estatisticamente associados com as internações por doenças respiratórias. No grupo de crianças, aumentos de um interquartil nas médias móveis de sete dias do $P M_{10}\left(56,5 \mu \mathrm{g} / \mathrm{m}^{3}\right) e$ de cinco dias do ozônio $\left(46,7 \mu \mathrm{g} / \mathrm{m}^{3}\right)$ levaram a aumentos nas internações hospitalares de 9,6\% (IC95\%: 3,0\%16,1\%) e 2,4\% (IC95\%: 0,1\%-4,7\%), respectivamente. Esforços para redução das concentrações dos poluentes do ar ainda precisam ser implementados para minimizar os seus efeitos adversos sobre crianças e adolescentes em Cubatão.

Poluição Atmosférica; Doenças Respiratórias; Internação Hospitalar; Criança; Adolescente

\section{Colaboradores}

R. Jasinski contribuiu com a concepção do estudo, análise e interpretação dos dados, redação e aprovação da versão final do artigo. L. A. A. Pereira participou da concepção do projeto, revisão crítica do artigo e aprovação da versão final. A. L. F. Braga participou da concepção do estudo, análise e interpretação dos dados, revisão crítica do manuscrito e aprovação da versão final.

\section{Referências}

1. Cançado JED, Braga ALF, Pereira LAA, Arbex MA, Saldiva PHN, Santos UPS. Repercussões clínicas da exposição à poluição atmosférica. J Bras Pneumol 2006; 32:5-11.

2. Samet J, Krewski D. Health effects associated with exposure to ambient air pollution. J Toxicol Environ Health A 2007; 70:227-42.

3. Moura M, Washington LJ, Gulnar ASM, Ponce de Leon A. Qualidade do ar e transtornos respiratórios agudos em crianças. Rev Saúde Pública 2008; 42:503-11.

4. Lin CA, Martins MA, Farhat SCL, Pope III CA, Conceição GMS, Anastácio VM, et al. Air pollution and respiratory illness of children in São Paulo, Brazil. Paediatr Perinatal Epidemiol 1999; 13:475-88.
5. Braga ALF, Saldiva PHN, Pereira LAA, Menezes JJC, Conceição GMS, Lin CA, et al. Health effects of air pollution exposure on children and adolescents in São Paulo, Brazil. Pediatr Pulmonol 2001;31:106-13.

6. Martins LC, Latorre MRDO, Saldiva PHN, Braga ALF. Air pollution and emergency room visits due to chronic lower respiratory diseases in the elderly: an ecological time-series study in São Paulo, Brazil. J Occup Environ Med 2002; 44:622-7.

7. Bakonyi SMC, Danni-Oliveira IM, Martins LC, Braga ALF. Poluição atmosférica e doenças respiratórias em crianças na cidade de Curitiba, PR. Rev Saúde Pública 2004; 38:695-700. 
8. Braga ALF, Pereira LAA, Procópio M, André PA, Saldiva PHN. Associação entre poluição atmosférica e doenças respiratórias e cardiovasculares na cidade de Itabira, Minas Gerais, Brasil. Cad Saúde Pública 2007; 23:570-8.

9. Pope III CA, Burnett RT, Thun MJ, Calle EE, Krewski D, Ito K, et al. Lung cancer, cardiopulmonary mortality, and long-term exposure to fine particulate air pollution. JAMA 2002; 287:1132-41.

10. Pope III CA, Burnett RT, Thruston GD, Thun MJ, Calle EE, Krewski D, et al. Cardiovascular mortality and long-term exposure to particulate air pollution: epidemiological evidence of general pathophysiological pathways of disease. Circulation 2004; 109:71-7.

11. Pope III CA. Mortality effects of longer term exposures to fine particulate air pollution: review of recent epidemiological evidence. Inhal Toxicol 2007; 9:33-8.

12. Bueno F, Fonseca A, Braga F, Miranda P. Qualidade do ar e internações por doenças respiratórias em crianças no município de Divinópolis, Estado de Minas Gerais. Acta Sci Health Sci 2010; 32:185-9.

13. Schwartz J. Air pollution and children's health. Pediatrics 2004; 113:1037-43.

14. Ostro B, Roth L, Malig B, Mart M. The effects of fine particle components on respiratory hospital admissions in children. Environ Health Perspect 2009; 117:475-80.

15. Martins LC, Latorre MRDO, Saldiva PHN, Braga ALF. Relação entre poluição atmosférica e atendimentos por infecção de vias aéreas superiores no município de São Paulo: avaliação do rodízio de veículos. Rev Bras Epidemiol 2001; 4:220-9.

16. Martins LC, Latorre MRDO, Cardoso MRA, Gonçalves FLT, Saldiva PHN, Braga ALF. Poluição atmosférica e atendimentos por pneumonia e gripe em São Paulo, Brasil. Rev Saúde Pública 2002; 36:88-94.

17. Martins MCH, Fatigati FL, Véspoli TC, Martins LC, Pereira LAA, Martins MA, et al. Influence of socioeconomic conditions on air pollution adverse health in elderly people: an analysis of six regions in São Paulo, Brazil. J Epidemiol Community Health 2004; 48:41-6.

18. Cakmak S, Dales RE, Vidal CB. Air pollution and mortality in Chile: susceptibility among the elderly. Environ Health Perspect 2007; 115:524-7.

19. Brook RD, Franklin B, Cascio W, Hong Y, Howard G, Lipsett M, et al. Air pollution and cardiovascular disease : a statement for healthcare professionals from the Expert Panel on Population and Prevention Science of the American Heart Association. Circulation 2004; 109:2655-71.

20. Dominici F, Peng RD, Bell ML, Pham L, McDermott A, Zeger SL, et al. Fine particulate air pollution and hospital admission for cardiovascular and respiratory diseases. JAMA 2006; 295:1127-33.

21. Nogueira JB. Poluição atmosférica e doenças cardiovasculares. Rev Port Cardiol 2009; 28:715-33.

22. Paulo S. Nossos doentes pneumopatas e a poluição atmosférica [Editorial]. J Bras Pneumol 2008; 34:1.

23. Almeida RS. A industrialização e a questão ambiental na Região Sudeste do Brasil. Caminhos de Geografia 2004; 4:53-66.
24. McCullagh P, Nelder JA. Generalized linear models. $2^{\text {nd }}$ Ed. London: Chapman \& Hall; 1989.

25. Green PJ, Silverman BW. Nonparametric regression and generalized linear models: a roughness penalty approach. London: Chapman \& Hall; 1994.

26. Braga ALF, Zanobetti A, Schwartz J. The lag structure between particulate air pollution and respiratory and cardiovascular deaths in ten US cities. J Occup Environ Med 2001; 43:927-33.

27. Braga ALF. Avaliação do efeito da poluição do ar sobre a saúde da população pediátrica da cidade de São Paulo e proposta de monitorização [Tese de Doutorado]. São Paulo: Faculdade de Medicina, Universidade de São Paulo; 1998.

28. Veras CMT, Martins MS. A confiabilidade dos dados nos formulários de Autorização de Internação Hospitalar (AIH), Rio de Janeiro, Brasil. Cad Saúde Pública 1994; 10:339-55.

29. Lin CA, Pereira LAA, Braga ALF, Conceição GMS, Kishi HS, Milani R, et al. Association between air pollution and ischemic cardiovascular emergency room visits. Environ Res 2003; 92:53-63.

30. Gouveia N, Mendonça GAS, Leon AP, Correia JEM, Junger WL, Freitas CU, et al. Poluição do ar e feitos na saúde nas populações de duas grandes metrópoles brasileiras. Epidemiol Serv Saúde 2003; 12:29-40.

31. Ignotti E, Hacon SS, Junger WL, Mourão D, Longo K, Freitas S, et al. Poluição do ar e admissões hospitalares por doenças respiratórias na Amazônia subequatorial: abordagem de séries temporais. Cad Saúde Pública 2010; 26:747-61.

32. Atkinson RW, Anderson HR, Sunyer J, Ayres J, Baccini M, Vonk JM, et al. Acute effects of particulate air pollution on respiratory admissions: results from APHEA 2 project. Air Pollution and Health: a European Approach. Am J Respir Crit Care Med 2001; 164:1860-6.

33. Andersen ZJ, Wahlin P, Raaschou-Nielsen O, Scheike T, Loft S. Ambient particle source apportionment and daily hospital admissions among children and elderly in Copenhagen. J Expo Sci Environ Epidemiol 2007; 17:625-36.

34. Freitas C, Bremner AS, Gouveia N, Pereira LAA, Saldiva PHN. Internações e óbitos e sua relação com a poluição atmosférica em São Paulo, 1993-1997. Rev Saúde Pública 2004; 38:751-7.

35. Alvim CG, Lasmar LMLBF. Saúde da criança e do adolescente: doenças respiratórias. Belo Horizonte: Editora Coopmed/Núcleo de Educação em Saúde Coletiva, Universidade Federal de Minas Gerais; 2009.

36. Gouveia N, Freitas C, Martins LC, Marcílio IO. Hospitalizações por causas respiratórias e cardiovasculares associadas à contaminação atmosférica no município de São Paulo, Brasil. Cad Saúde Pública 2006; 22:2669-77.

37. Farhat SCL, Paulo RLP, Shimoda TM, Conceição GMS, Lin CA, Braga ALF, et al. Effect of air pollution on pediatric respiratory emergency room visits and hospital admissions. Braz J Med Biol Res $2005 ; 38: 227-35$. 
38. Burnett RT, Smith-Doirom M, Stieb D, Raizenne ME, Brook JR, Dales RE, et al. Association between ozone and hospitalization for acute respiratory diseases in children less than 2 years of age. Am J Epidemiol 2001; 153:444-52.

39. Lin M, Stieb DM, Chen Y. Coarse particulate matter and hospitalization for respiratory infections in children younger than 15 years in Toronto: a casecrossover analysis. Pediatrics 2005; 116:235-40.

40. Lin S, Bell EM, Liu W, Walker RJ, Kim NK, Hwang SA. Ambient ozone concentration and hospital admissions due to childhood respiratory diseases in New York State, 1991-2001. Environ Res 2008; 108:42-7.
41. Ciencewicki J, Brighton L, Wu W, Madden M, Jaspers I. Diesel exhaust enhances virus- and poly (I:C)-induced Toll-like receptor 3 expression and signaling in respiratory epithelial cells. Am J Physiol Lung Cell Mol Physiol 2006; 290:1154-63.

42. Souza MB, Saldiva PHN, Pope III CA, Capelozzi VL. Respiratory changes due to long-term exposure to urban levels of air pollution - a histopathologic study in humans. Chest 1998; 113:1312-8.

43. Chauhan AJ, Johnston SL. Air pollution and infection in respiratory illness. Br Med Bull 2003; 68: 95-112.

Recebido em 28/Mar/2011

Versão final reapresentada em 17/Ago/2011

Aprovado em 29/Ago/2011 